\title{
Impacto da adubação orgânica sobre a incidência de tripes em cebola
}

\author{
Paulo Antônio de S. Gonçalves ${ }^{1}$; Carlos Roberto Sousa e Silva ${ }^{2}$ \\ ${ }^{1}$ EPAGRI, EE Ituporanga, C. Postal 121, 88400-000, Ituporanga-SC (Bolsista Embrapa); E-mail: pasg@epagri.rct-sc.br ; ${ }^{2}$ UFSCar, \\ Depto. Ecologia e Biologia Evolutiva, C. Postal 676, 13565-905, São Carlos-SP. E-mail: dcrs@power.ufscar.br
}

\begin{abstract}
RESUMO
Analisou-se a relação entre adubação orgânica e a incidência de Thrips tabaci Lind. em cebola (Allium cepa L), na EE de Ituporanga, entre agosto e dezembro de 1998. Os tratamentos foram determinados de acordo com a necessidade de $\mathrm{N}$ para a cultura pela análise de solo. Empregou-se como fonte orgânica diversos adubos fornecendo $75 \mathrm{Kg} /$ ha de N (esterco suíno; adubo Barriga Verde ${ }^{\circledR}$ proveniente de esterco de aves; composto orgânico; esterco de peru; húmus); $37,5 \mathrm{Kg} / \mathrm{ha}$ de $\mathrm{N}$ (metade da dose normal com esterco de suíno); as testemunhas foram adubação mineral fornecendo $30-120-60 \mathrm{~kg} / \mathrm{ha}$ de N-P $\mathrm{O}_{5}-\mathrm{K}_{2} \mathrm{O}$ e o dobro da dose $\left(60-240-120 \mathrm{~kg} / \mathrm{ha}\right.$ de N-P $\mathrm{O}_{5}-$ $\mathrm{K}_{2} \mathrm{O}$ ); e testemunha sem adubação. Nenhum tratamento apresentou incidência de T. tabaci superior à testemunha sem adubo. A adubação mineral em relação à orgânica não favoreceu significativamente a incidência de T. tabaci. O processo de conversão do manejo do solo da área experimental de convencional para orgânico pode ter favorecido a infestação similar do inseto entre tratamentos. No período de maior incidência de $T$. tabaci, a relação com nutrientes foi descrita por um modelo envolvendo $\mathrm{K} / \mathrm{Zn}, \mathrm{B}$ e $\mathrm{N}$ de maneira positiva. A correlação entre nutrientes e T. tabaci não foi linear na maioria das avaliações. A adubação orgânica pode substituir a adubação mineral na cultura da cebola, pois foi possível atingir níveis de produtividade similares para ambos tratamentos.
\end{abstract}

Palavras-chave: Allium cepa, Thrips tabaci, inseto, nutriente, agroecologia.

\begin{abstract}
Impact of the organic fertilization on onion thrips incidence

The impact of organic fertilization on the incidence of thrips (Thrips tabaci Lind.) on onion was evaluated at Ituporanga Experimental Station in Santa Catarina State, Brazil, from August to December, 1998. The treatments using organic fertilization were based on the $\mathrm{N}$ needs determined by previous soil analysis. Using different organic sources were $75.0 \mathrm{Kg} / \mathrm{ha}$ of $\mathrm{N}$ (swine manure; Barriga Verde ${ }^{\circledR}$ poultry manure; compost; turkey manure; humus); $37.5 \mathrm{Kg} / \mathrm{ha}$ of $\mathrm{N}$ (swine manure half regular rate); two chemically fertilized controls with $30-120-60 \mathrm{Kg} /$ ha of N- $\mathrm{P}_{2} \mathrm{O}_{5}-\mathrm{K}_{2} \mathrm{O}$ and double rate $\left(60-240-120 \mathrm{Kg} / \mathrm{ha}\right.$ of $\left.\mathrm{N}-\mathrm{P}_{2} \mathrm{O}_{5}-\mathrm{K}_{2} \mathrm{O}\right)$; and finally the unfertilized control. The incidence of thrips was similar between treatments with fertilized and no fertilized check. Treatments with mineral fertilization presented the same incidence of thrips when compared to organic fertilization. During severe infestation period, the correlation between the use of mineral nutrients and the T. tabaci incidence was positive and was described by a model involving the levels of $\mathrm{K} / \mathrm{Zn}, \mathrm{B}$ and $\mathrm{N}$. The correlation of nutrient in the leaves and the infestation of thrips on onion was not linear. The organic fertilization can substitute the mineral one on onion crops, without reducing yield.
\end{abstract}

Keywords: Allium cepa, Thrips tabaci, insect, nutrient, agroecology.

(Recebido para publicação em 18 de março de 2002 e aceito em 04 de julho de 2003)

$\mathrm{O}$ tripes (Thrips tabaci Lindeman (Thysanoptera: Thripidae) ) é a principal praga da cebola no Brasil (Gallo et al., 1988). Em Santa Catarina, o manejo fitossanitário da cultura após o transplantio tem-se caracterizado principalmente pela aplicação de agrotóxicos para o seu controle. Gonçalves (1996) observou que a aplicação de inseticidas organofosforados e piretróides para controle de tripes tem sido exagerada em dose e número de aplicações com prejuízos à saúde do agricultor e ambiente.

A redução da produtividade causada por T. tabaci em cebola pode ser além de 50\% em sistema de agricultura convencional (Saini et al., 1989). Porém, em recentes trabalhos, tem-se observado que, em solos sob plantio direto e altos níveis de matéria orgânica, minimizam-se as perdas em produtivi- dade independente do controle químico do inseto (Gonçalves, 1998).

A fertilização de plantas apresenta efeitos positivos e negativos na incidência de pragas e doenças (Maxwell, 1972, citado por Bortolli \& Maia, 1994; Rodriguez, 1972, citado por Bortolli \& Maia, 1994; Primavesi, 1988; Patriquin et al., 1993; Bortolli \& Maia, 1994). Enquanto Chaboussou (1987) e Primavesi (1988) ressaltam a importância do equilíbrio nutricional para evitarse doenças e pragas, Maxwell (1972), citado por Bortolli \& Maia (1994) considerou que os insetos ficam com menor vigor devido à nutrição inadequada das plantas, e consequentemente mais vulneráveis à ação dos produtos químicos e biológicos usados no seu controle. As demais práticas de manejo de agroecossistemas em sistema convencional tais como a aplicação de agrotóxicos, a mecanização excessiva do solo com máquinas, têm efeito direto e indireto no empobrecimento da fauna de solo responsável pela ciclagem de nutrientes (Matson et al. 1997; Altieri \& Nicholls 1999). As plantas produzidas em ambientes com excesso de agroquímicos apresentam-se desequilibradas nutricionalmente e vulneráveis ao ataque de doenças e pragas (Primavesi, 1988; Altieri \& Nicholls, 1999). O manejo do solo com práticas que não agridam a biota e favoreçam a ciclagem de nutrientes é fundamental para obtenção de plantas saudáveis, tais como: redução da mecanização do solo (plantio direto ou cultivo mínimo); uso preferencial de adubos verdes; plantas de cobertura; estercos; compostos (Yepsen Jr., 1977; Primavesi, 1988; Altieri, 1991; Monegat, 1991; Patriquin et al. 1993; Altieri, 1994; Matson et al., 
1997; Vandermeer, 1995; Altieri \& Nicholls, 1999).

A EPAGRI atualmente fomenta trabalhos na área de agroecologia com o intuito de reduzir a utilização de agroquímicos nesse estado, bem como gerar um sistema agrícola produtivo e sustentável. O presente trabalho foi elaborado para contribuir na geração de informações para sedimentar a agroecologia no estado de Santa Catarina e para avaliar o efeito das adubações mineral e orgânica sobre a ocorrência do tripes e no impacto dos diferentes tratamentos sobre a produtividade da cultura da cebola.

\section{MATERIAL E MÉTODOS}

O experimento foi conduzido na Estação Experimental de Ituporanga em um Cambissolo Húmico distrófico álico, empregando a cultivar Crioula.

O experimento foi realizado de 28/ 08 (transplante) a 16/12/1998 (colheita, duração do ciclo de 110 dias), implantado em delineamento de blocos ao acaso com quatro repetições. O tamanho da parcela foi de 2,8 x 3,0 m e espaçamento de $40 \times 10 \mathrm{~cm}$, totalizando 210 plantas/ parcela. As parcelas foram distanciadas entre si de $1 \mathrm{~m}$. O preparo do solo foi realizado sob plantio direto em palhada de centeio (Secale cereale L.), semeado anteriormente no mês de maio. Os tratamentos foram: 1) $15743,6 \mathrm{~kg} / \mathrm{ha} \mathrm{de}$ esterco de suíno, 2) $7871,8 \mathrm{~kg} / \mathrm{ha}$ de esterco de suíno (metade da dose recomendada pela análise de solo) , 3) 6023,6 $\mathrm{kg} /$ ha de esterco Barriga Verde $\AA$ (aves), 4) $20299,9 \mathrm{~kg} / \mathrm{ha}$ de composto orgânico, 5) $6393,5 \mathrm{~kg} / \mathrm{ha}$ de esterco de peru, 6) $6516,4 \mathrm{~kg} / \mathrm{ha}$ de húmus, 7) $600,0 \mathrm{~kg} /$ ha de NPK 5-20-10, 8) 1200,0 kg/ha de NPK 5-20-10, 9) testemunha sem adubação. Os tratamentos com fontes orgânicas forneceram a necessidade de $\mathrm{N}$ para a cultura $(75 \mathrm{~kg} / \mathrm{ha})$, exceto o número 2 , que correspondeu à metade da dose $(37,5 \mathrm{~kg} / \mathrm{ha})$. Os tratamentos 7 e 8 corresponderam a testemunhas com adubação mineral com fornecimento de NPK uma e duas vezes respectivamente o recomendado para a cultura de acordo com a análise de solo. A análise de solo apresentou : pH (água), 6,3; pH (SMP), 6,1; P, 25,5 mg.dm ${ }^{-3}$ K, 40 mg.dm ${ }^{-3} ;$ matéria orgânica, 50 g.dm ${ }^{-3} ; \mathrm{Al}$ $0,0 \mathrm{mmol} \cdot \mathrm{dm}^{-3} ; \mathrm{Ca}, 74 \mathrm{mmol} \cdot \mathrm{dm}^{-3} ; \mathrm{Mg}$, $38 \mathrm{mmol} \cdot \mathrm{dm}^{-3}$; argila, $330 \mathrm{~g} \cdot \mathrm{dm}^{-3}$. Como o nitrogênio é apontado como causador de desequilíbrios nutricionais e favorecer a incidência de doenças e pragas (Chaboussou, 1987; Singh \& Agarwal, 1983, citados por Bortolli \& Maia, 1994; Schuch et al., 1998), a determinação da quantidade de adubos orgânicos foi baseada na necessidade deste nutriente para a cultura (segundo Comissão de Fertilidade do Solo RS/SC, 1994). A fórmula utilizada para calcular a dose de esterco/ha foi

Quantidade de esterco/ha $=(100000$ x N)/(MS x NMS) x 0,5;

onde $\mathrm{N}$ é a quantidade de nitrogênio em $\mathrm{kg} / \mathrm{ha}$ necessário para a cultura da cebola; MS é o teor de matéria seca do esterco em porcentagem; NMS quantidade de nitrogênio por $\mathrm{kg}$ de matéria seca do esterco; e 0,5 é o índice de liberação de nitrogênio pelo esterco $(50 \%$ no primeiro ano de aplicação, de acordo com Comissão de Fertilidade do Solo RS/SC, 1994). A análise de nutrientes dos adubos orgânicos foi realizada pelo Laboratório de Fisiologia e Nutrição Vegetal da Estação Experimental de Caçador, SC, EPAGRI, com os seguintes resultados para matéria seca (MS, em g. $\mathrm{dm}^{-3}$ ), e níveis de nutrientes em $\mathrm{g} / \mathrm{kg}^{1}$ e $\mathrm{mg} / \mathrm{kg}^{2}$ de MS respectivamente para esterco de suíno, esterco Barriga Verde ${ }^{\circledR}$ (aves), composto orgânico, húmus, esterco de peru: MS $(27,3 ; 93,4 ; 36,4$; $61,2 ; 79,8), \mathrm{N}^{1}(34,9 ; 26,5 ; 20,3 ; 37,6$; $29,4), \mathrm{P}^{1}(23,6 ; 15,8 ; 14,7 ; 26,6 ; 20,8)$, $\mathrm{K}^{1}(1,8 ; 15,0 ; 3,4 ; 7,2 ; 25,4), \mathrm{Ca}^{1}(52,8$; 64,6; 29,0; 58,0; 31,0), $\operatorname{Mg}^{1}(4,2 ; 15,6$; $5,0 ; 6,4 ; 8,2), \mathrm{Fe}^{2}(3822,0 ; 1074,0$; $8686,0 ; 6008,0 ; 7230,0), \mathrm{Mn}^{2}(368,0$; $636,0 ; 318,0 ; 634,0 ; 574,0), \mathrm{Zn}^{2}(650,0$; 298,0; 244,0; 990,0; 468,0), $\mathrm{Cu}^{2}$ (596,0; $108,0 ; 232,0 ; 734,0 ; 112,0), \mathrm{B}^{2}(31,0$; $53,0 ; 34,0 ; 42,0 ; 58,0)$.

O composto orgânico foi proveniente de resíduos culturais de cebola, esterco suíno, capim cameroon (Pennisetum clandestinum Hochst. ex. Chiov). $\mathrm{O}$ húmus foi obtido de esterco de suíno em minhocário. Os adubos orgânicos foram distribuídos manualmente e espalhados sobre a superfície do solo, enquanto as fontes minerais foram incorporadas com microtrator adaptado para plantio direto.
A amostragem de tripes foi realizada a campo pela contagem semanal de ninfas com auxílio de lupa manual (aumento de $3 \mathrm{x}$ ), em todas as folhas em 5 plantas/parcela escolhidas ao acaso. As datas de avaliação foram 14/10 (47 dias após transplante, DAT), 21/10 (54 DAT), 28/10 (61 DAT), 04/11 (68 DAT), 11/11 (75 DAT), 19/11 (83 DAT), 25/11 (89 DAT) e 02/12/1998 (96 DAT).

A análise foliar de nutrientes foi realizada em amostras de cinco plantas por parcela, submetidas a secagem em estufa a $60^{\circ} \mathrm{C}$, e posteriormente enviadas para o Laboratório de Fisiologia e Nutrição Vegetal da EE de Caçador. As datas de coleta de amostras e respectivos dias após transplante (DAT) foram: 14/10 (47 DAT), 11/11 (75 DAT) e 02/ 12/1998 (96 DAT).

$\mathrm{O}$ experimento não foi submetido à irrigação, com a finalidade de evitar-se a influência de remoção mecânica do inseto pela água.

A amostragem para produtividade realizou-se pela colheita de 60 bulbos ao acaso por parcela. Os bulbos foram classificados pelo diâmetro de acordo com aceitação para o mercado (classe 1 $\leq 3,5 \mathrm{~cm}$; classe $2>3,5$ até $5 \mathrm{~cm}$; classe $3>5$ até $7 \mathrm{~cm}$; classe $4>7$ até $9 \mathrm{~cm}$, classe $5>9 \mathrm{~cm}$ ). Apenas a produtividade comercial (bulbos de classes 2 a 5) foi considerada para avaliação.

O nível de dano econômico (NDE) foi estabelecido de acordo com Gonçalves (1997), sendo considerado 15 ninfas/ planta antes da bullbificação e 30 ninfas/ planta após esta fase.

Os dados para análise estatística foram previamente submetidos ao teste de Bartlett (através do programa para análise estatística INSTAT®, 1993) a fim de verificar a homogeneidade de variâncias e definir a aplicação de métodos paramétricos ou não paramétricos. Após a transformação de dados foi possível realizar a análise de variância paramétrica. $\mathrm{O}$ número médio de ninfas de tripes foi transformado para $\log (\mathrm{x}+$ $0,5)$. As médias foram comparadas pelo teste de Tukey a 5\% de probabilidade (programa para análise estatística $\mathrm{SAS} \AA$, 1996). O nível foliar de nutrientes e produtividade foram submetidos à análise de variância sem transformação de dados. O esquema de análise de variância 
Tabela 1. Número médio de ninfas de tripes, T. tabaci, produtividade comercial média e peso médio de bulbos de cebola, cultivar Crioula, sob adubações mineral e orgânica. Ituporanga, SC, EPAGRI, 1998.

\begin{tabular}{lcccc}
\hline \multicolumn{1}{c}{ Tratamentos } & $\begin{array}{c}\text { Doses de } \\
\text { nutrientes } \\
(\mathbf{k g} / \mathbf{h a})\end{array}$ & Ninfas de tripes & $\begin{array}{c}\text { Produtividade } \\
\mathbf{( t / h a )}\end{array}$ & $\begin{array}{c}\text { Peso médio de } \\
\text { bulbos } \mathbf{( g )}\end{array}$ \\
\hline N (esterco de suíno) & 75,0 & $17,5 \mathrm{a}$ & $12,9 \mathrm{a}$ & $58,1 \mathrm{abc}$ \\
N (esterco de suíno) & 37,5 & $17,8 \mathrm{a}$ & $12,2 \mathrm{a}$ & $54,4 \mathrm{bc}$ \\
N (esterco Barriga Verde ${ }^{\circ}$, aves) & 75,0 & $19,8 \mathrm{a}$ & $13,9 \mathrm{a}$ & $62,8 \mathrm{abc}$ \\
N (composto orgânico) & 75,0 & $17,7 \mathrm{a}$ & $11,6 \mathrm{a}$ & $52,2 \quad \mathrm{c}$ \\
N (esterco de peru) & 75,0 & $16,7 \mathrm{a}$ & $16,9 \mathrm{a}$ & $71,9 \mathrm{a}$ \\
N (húmus) & 75,0 & $17,1 \mathrm{a}$ & $12,0 \mathrm{a}$ & $55,6 \mathrm{bc}$ \\
N P K (5-20-10) & $30-120-60$ & $19,8 \mathrm{a}$ & $15,6 \mathrm{a}$ & $68,8 \mathrm{ab}$ \\
N P K (5-20-10) & $60-240-120$ & $21,0 \mathrm{a}$ & $15,7 \mathrm{a}$ & $69,2 \mathrm{ab}$ \\
Testemunha (sem adubação) & & $19,3 \mathrm{a}$ & $12,0 \mathrm{a}$ & $55,8 \mathrm{abc}$ \\
\hline CV\% & & 20,6 & 17,6 & 11,0 \\
\hline
\end{tabular}

Médias na vertical, seguidas da mesma letra não diferem entre si pelo teste de Tukey a 5\% de probabilidade.

para análise das variáveis número médio de ninfas de tripes e nível foliar de nutrientes foi o de parcelas subdivididas no tempo com tratamentos como parcelas e datas de avaliação como subparcelas. Os coeficientes de correlação de Spearman (r) e de determinação $\left(\mathrm{r}^{2}\right)$ e a análise de regressão linear múltipla, procedimento "stepwise" do programa estatítico SAS ${ }^{\circledR}$ (1996), foram aplicados entre a incidência de tripes, e nível foliar de nutrientes.

\section{RESULTADOS E DISCUSSÃO}

$\mathrm{O}$ número médio de ninfas de $T$. tabaci não diferiu entre os tratamentos (Tabela 1). Portanto, como constatado por Marzo et al. (1997), Bastos et al. (1998), Osuna et al. (1989), Silva et al. (1998), a utilização de adubação mineral não necessariamente favorece a maior densidade populacional de herbívoros, pois o efeito pode ser não significativo ou até mesmo inferior ao adubo orgânico.

O nível de nutrientes foliares diferiu entre tratamentos apenas para $\mathrm{N} \mathrm{e} \mathrm{K}$ (Tabela 2). $\mathrm{O}$ teor de $\mathrm{N}$ foi superior para esterco de suíno, dose normal, em relação ao NPK dose dupla, porém estes não diferiram dos demais tratamentos. $\mathrm{O}$ teor de $\mathrm{K}$ no composto orgânico foi superior ao do esterco de suíno na metade da dose normal, porém foi semelhante aos demais. Portanto, as fontes de adubação utilizadas, com dosagens de adubo entre normal até no máximo o dobro recomendado pela análise do solo, não proporcionaram diferenças significativas nos teores de nutrientes foliares na maioria das avaliações que pudessem refletir na incidência de tripes em cebola. Deve-se considerar, que a área experimental estava em processo de conversão para manejo orgânico (solo sob sistema de plantio direto, uso de adubos verdes e estercos, e ausência de adubação mineral solúvel) há dois anos, o que pode ter favorecido a incidência similar do inseto entre tratamentos. Porém, segundo Phelan (1997), plantas cultivadas em solos sob manejo orgânico têm uma tendência em apresentar menores níveis populacionais de insetos.

Os coeficientes de correlação linear (r) e de determinação $\left(\mathrm{r}^{2} \%\right)$ entre nutrientes e inseto não foram significativos na maioria das avaliações, exceto de maneira significativa a $1 \%$ para a ocorrência de tripes no fim do ciclo da cultura (02/ 12/1998) para $\mathrm{K} / \mathrm{Cu}\left(\mathrm{r}=-0,49^{* *}\right)$ e nas relações inversas de alguns nutrientes com $\mathrm{Mn}\left(\mathrm{N} / \mathrm{Mn}, \mathrm{r}=0,44^{* *} ; \mathrm{P} / \mathrm{Mn}\right.$, $\left.\mathrm{r}=0,46^{* *} ; \mathrm{Mg} / \mathrm{Mn}, \mathrm{r}=0,45^{* *}\right)$. Este resultado sugere que excesso dos nutrientes N, P e Mg em relação a Mn favoreceu a ocorrência de tripes, enquanto o aumento de $\mathrm{K}$ em relação a $\mathrm{Cu}$ diminuiu a incidência do inseto. Convém ressaltar, que tais relações foram verificados no período de maturação fisioló- gica da cultura, quando a presença do inseto entra naturalmente em declínio e não provoca maiores danos à cultura; $\mathrm{o}$ nível de dano econômico (NDE), nesta fase é de 30 ninfas/planta, segundo Gonçalves (1997). O potássio como indutor de resistência a insetos e patógenos foi relatado por Chaboussou (1987), Primavesi (1988) e Bortolli \& Maia (1994); e Mn por Chaboussou (1987). $\mathrm{O}$ valor de $\mathrm{r}$ foi significativo a $5 \%$ para os seguintes nutrientes: em 11/11 para $\mathrm{K} / \mathrm{Zn}(\mathrm{r}=0,36)$, e em $2 / 12$ para $\mathrm{K}(\mathrm{r}=-0,38), \mathrm{K} / \mathrm{Mg}(\mathrm{r}=-0,34)$, $\mathrm{K} / \mathrm{B}(\mathrm{r}=-0,40), \mathrm{Mn} / \mathrm{Zn}(\mathrm{r}=-0,35)$, $\mathrm{Cu}(\mathrm{r}=0,33), \mathrm{N} / \mathrm{K}(\mathrm{r}=0,33), \mathrm{P} / \mathrm{K}$ $(r=0,38), \mathrm{Ca} / \mathrm{Mn}(\mathrm{r}=0,35)$. Dentre estas relações o excesso de $\mathrm{N}$ em relação a $\mathrm{K}$ foi citado por favorecer o desenvolvimento de afídeos em quiabeiro (Sampaio et al. 1998a, b), e as proporções entre $\mathrm{K} / \mathrm{Mg}, \mathrm{K} / \mathrm{B}, \mathrm{Ca} / \mathrm{Mn}, \mathrm{N} / \mathrm{P} / \mathrm{K}$ foram citadas como importantes na manutenção do equilíbrio nutricional de plantas por Primavesi (1988). Convém ressaltar, que em 11/11, na semana anterior ao pico populacional do inseto houve relação significativa positiva para $\mathrm{K} / \mathrm{Zn}$, o que sugere que nesta fase o excesso de $\mathrm{K}$ em relação a $Z n$ favoreceu a infestação de tripes. Os resultados significativos observados em 2/12 ocorreram na fase de maturação fisiológica da cultura, quando a população de tripes estava abaixo do NDE (de acordo com Gonçalves, 1997, nesta fase o NDE é 
Tabela 2. Níveis de nutrientes em folhas de cebola sob fontes de adubação mineral e orgânica. Ituporanga, SC, EPAGRI, 1998.

\begin{tabular}{|c|c|c|c|c|c|c|c|c|c|c|c|}
\hline \multirow{2}{*}{ Tratamentos } & \multirow{2}{*}{$\begin{array}{c}\text { Doses de } \\
\text { nutrientes } \\
\text { (kg/ha }\end{array}$} & \multicolumn{10}{|c|}{ Nutrientes } \\
\hline & & $\mathbf{N}^{1}$ & $\mathbf{P}^{1}$ & $\mathrm{~K}^{1}$ & $\mathrm{Ca}^{1}$ & $M^{1}$ & $\mathrm{Fe}^{2}$ & $M n^{2}$ & $Z^{2}$ & $\mathrm{Cu}^{2}$ & $B^{2}$ \\
\hline $\mathrm{N}$ (esterco de suíno) & 75,0 & $32,5 \mathrm{a}$ & $4,0 \mathrm{a}$ & $24,2 a b$ & $11,5 \mathrm{a}$ & $4,3 a$ & $158,8 \mathrm{a}$ & $19,6 \mathrm{a}$ & $13,5 \mathrm{a}$ & $7,0 \mathrm{a}$ & $22,0 \mathrm{a}$ \\
\hline N (esterco de suíno) & 37,5 & $31,9 a b$ & $4,2 \mathrm{a}$ & $21,3 b$ & $11,6 \mathrm{a}$ & $4,4 \mathrm{a}$ & $206,4 \mathrm{a}$ & $22,3 \mathrm{a}$ & $13,8 \mathrm{a}$ & $7,5 \mathrm{a}$ & $23,0 \mathrm{a}$ \\
\hline $\begin{array}{l}\text { N (esterco Barriga } \\
\text { Verde } \AA \text {, aves) }\end{array}$ & 75,0 & $31,9 a b$ & $4,5 \mathrm{a}$ & $26,8 a b$ & $11,0 \mathrm{a}$ & $4,1 \mathrm{a}$ & 177,7 a & $24,8 \mathrm{a}$ & $15,6 a$ & 8,6 a & $23,4 \mathrm{a}$ \\
\hline $\begin{array}{l}\mathrm{N} \text { (composto } \\
\text { orgânico) }\end{array}$ & 75,0 & $31,8 a b$ & $4,2 \mathrm{a}$ & $28,1 \mathrm{a}$ & 11,8 a & $4,4 \mathrm{a}$ & 226,6 a & $25,8 \mathrm{a}$ & $15,3 a$ & 9,6 a & $21,2 \mathrm{a}$ \\
\hline $\mathrm{N}$ (esterco de peru) & 75,0 & $31,0 a b$ & $4,1 \mathrm{a}$ & $22,7 a b$ & $11,0 \mathrm{a}$ & $4,1 \mathrm{a}$ & $181,4 \mathrm{a}$ & $27,0 \mathrm{a}$ & $13,5 \mathrm{a}$ & $7,8 \mathrm{a}$ & $22,7 \mathrm{a}$ \\
\hline $\mathrm{N}$ (húmus) & 75,0 & $30,3 a b$ & $4,0 \mathrm{a}$ & $21,9 a b$ & $11,4 \mathrm{a}$ & $4,2 \mathrm{a}$ & $230,7 \mathrm{a}$ & $24,6 \mathrm{a}$ & $15,8 \mathrm{a}$ & $7,7 \mathrm{a}$ & $24,3 \mathrm{a}$ \\
\hline N P K (5-20-10) & $30-120-60$ & $30,7 a b$ & $4,0 \mathrm{a}$ & $27,2 a b$ & $10,8 \mathrm{a}$ & 3,8 a & $162,5 \mathrm{a}$ & 26,9 a & $12,3 a$ & $7,6 \mathrm{a}$ & $21,3 \mathrm{a}$ \\
\hline N P K (5-20-10) & $60-240-120$ & $30,1 \quad b$ & $4,1 \mathrm{a}$ & $26,3 a b$ & $11,2 \mathrm{a}$ & $4,2 \mathrm{a}$ & $156,0 \mathrm{a}$ & $29,3 \mathrm{a}$ & $11,5 \mathrm{a}$ & $8,3 a$ & $20,6 a$ \\
\hline $\begin{array}{l}\text { Testemunha } \\
\text { (sem adubação) }\end{array}$ & & $31,1 \mathrm{ab}$ & $4,0 \mathrm{a}$ & $24,1 a b$ & $11,3 \mathrm{a}$ & $4,5 \mathrm{a}$ & 174,6 a & $20,8 \mathrm{a}$ & 14,4 a & $10,5 a$ & 20,9 a \\
\hline$\overline{C V} \%$ & & 5,8 & 15,1 & 20,2 & 16,1 & 14,7 & 48,6 & 38,5 & 35,5 & 60,9 & 17,8 \\
\hline
\end{tabular}

Médias na vertical, seguidas da mesma letra não diferem entre si pelo teste de Tukey a $5 \%$ de probabilidade, 1 e 2, respectivamente níveis foliares de nutrientes em g.dm ${ }^{-3} \mathrm{e} \mathrm{mg.dm}{ }^{-3}$.

de 30 ninfas/planta), portanto o inseto não interferiria na produtividade.

No presente trabalho a relação entre nutrientes e inseto geralmente não foi linear. Este tipo de relação entre nitrogênio e artrópodos já havia sido constatada anteriormente (White, 1984; Popp et al., 1986; Brewer et al., 1987; Rutz et al., 1990; Bethke et al., 1998; Joern \& Behmer, 1998). Portanto, o nitrogênio de maneira isolada não apresentou efeito significativo sobre o inseto, mas apenas nas relações com alguns nutrientes como apontado acima.

A análise de regressão múltipla apresentou as seguintes equações para relação entre número médio de ninfas de tripes (y) e nutrientes: para início da infestação do inseto em 14/10 com população abaixo do NDE, considerado como 15 ninfas/planta, de acordo com Gonçalves (1997), $\mathrm{y}=+10,94-0,04 \times \mathrm{P} / \mathrm{B}$, $\left(r^{2}=10,16 \%\right)$; em $11 / 11$, uma semana antes do pico populacional de tripes, com todos tratamentos acima do NDE, $\mathrm{y}=-51,35+0,008 \times \mathrm{K} / \mathrm{Zn}+0,89 \mathrm{xB}$ $+1,63 \times \mathrm{xN},\left(\mathrm{r}^{2}=33,2 \%\right)$; e no declínio populacional em $02 / 12$, com todos tratamentos abaixo do NDE, $\mathrm{y}=+21,32$ $0,004 \times K / \mathrm{Cu}+1,48 \times \mathrm{Mn} / \mathrm{Cu}+0,059 \times \mathrm{P} /$ $\mathrm{Mn}-0,38 \mathrm{xFe} / \mathrm{B},\left(\mathrm{r}^{2}=53,19 \%\right)$.

A generalização de $\mathrm{N}$ como causador de desequilíbrio nutricional e favorecer a ocorrência de pragas não foi constatada como notado por Bortolli \&
Maia (1994) e Rodriguez (1972) citados por estes autores, pois a relação isolada de N não apresentou valores significativos para incidência de tripes. Bortolli \& Maia (1994) relataram que embora a maioria dos trabalhos apontem para o efeito positivo de $\mathrm{N}$ no desenvolvimento de pragas, há resultados que indicam o contrário. $\mathrm{O}$ efeito negativo de $\mathrm{N}$ no desenvolvimento de insetos também pode ocorrer (Salas et al., 1990; Rutz et al., 1990), bem como o efeito não significativo (Power, 1989; McCullough \& Kulman,1991; Blua \& Toscano, 1994). Os resultados referentes ao nitrogênio contrastam com os de Schuch et al. (1998), que constataram que a infestação de tripes em crisântemo foi favorecida por altos níveis de adubação nitrogenada. $\mathrm{O}$ efeito positivo de $\mathrm{N}$ para incidência de tripes foi verificado de maneira significativa para o nutriente apenas nas relações N/Mn e $\mathrm{N} / \mathrm{K}$. Os resultados foram similares aos obtidos por Vos \& Frinking (1997) com efeito não significativo de $\mathrm{N}$ sobre tripes, T. parvispinus, em pimenta, Capsicum spp., porém a relação positiva de P sobre o inseto encontrada por estes autores não foi constatada, pois a relação foi significativamente positiva apenas para relação $\mathrm{P} / \mathrm{K}$ em 02/12.

A produtividade comercial foi similar entre tratamentos (Tabela 1). O peso médio de bulbos foi superior para ester- co de peru em relação ao composto orgânico, esterco de suíno metade da dose normal e húmus (Tabela 1). $\mathrm{O}$ adubo mineral NPK em ambas dosagens também apresentou peso médio de bulbos superior ao composto orgânico. Porém, nenhum tratamento proporcionou incremento significativo no peso médio de bulbos em relação a testemunha sem adubo (Tabela 1). Como os níveis de produtividade comercial e peso médio de bulbos obtidos por todas as fontes de N, com exceção do composto orgânico, não diferiram daqueles observados pela adubação mineral, sugere-se ser possível substituir a adubação de origem mineral pela orgânica.

\section{AGRADECIMENTOS}

Ao Dr. Odo Primavesi (Embrapa Pecuária Sudeste) e Dra. Márcia Mondardo Spengler (EPAGRI, EE Caçador) pelos esclarecimentos na área de análise estatística. Ao pesquisador Clori Basso (EPAGRI, EE Caçador) pelas análises de nutrientes. Ao técnico agrícola Marcelo Pitz e sua equipe pelo apoio na condução do trabalho.

\section{LITERATURA CITADA}

ALTIERI, M.A. How best can we use biodiversity in agroecosystems? Outlook on Agriculture, v. 20, n. 1, p. 15-23, 1991. 
ALTIERI, M.A. Bases agroecologicas para una producción agricola sustentable. Agricultura Tecnica, v. 54, n. 4, p. 371-386, 1994.

ALTIERI, M.A.;NICHOLLS, C.I. Ecologically based pest management: a key pathway to achieving agroecosystem health. In: NICHOLLS, C.I.; GARCIA, M.A.; ALTIERI, M.A., Comp. Curso de agroecologia, Workshop sobre agroecologia e desenvolvimento sustentável. Campinas: UNICAMP, 1999. v. 2, n .6.

BASTOS, C.S.; PICANÇO, M.; GALVÃO, J.C.C.; SUINAGA, F.A.; GORING, A.H.R.; MOURA, M.F. Efeito da adubação orgânica e mineral na população de pragas do feijão. In: CONGRESSO BRASILEIRO DE ENTOMOLOGIA, 17., 1998, Rio de Janeiro. Resumos... Rio de Janeiro: SEB/UFRRJ, 1998. p. 418.

BETHKE, J.A.; REDAK, R.A.; SCHUCH, U.K. Melon aphid performance on chrysanthemum as mediated by cultivar, and differential levels of fertilization and irrigation. Entomologia Experimentalis et Applicata, v. 88, n. 1, p. 41-47, 1998.

BLUA, M.J.; TOSCANO, N.C. Bemisia argentifolii (Homoptera: Aleyrodidae) development and honeydew production as a function of cotton nitrogen status. Environmental Entomology, v. 23, n. 2, p. 316-321, 1994.

BORTOLLI, S.A.; MAIA, I.G. Influência da aplicação de fertilizantes na ocorrência de pragas. In: SÁ, M.E.; BUZZETI, S., coord. Importância da adubação na qualidade dos produtos agrícolas. São Paulo: Icone, 1994. p. 53-63.

BREWER, J.W.; O’NEILL, K.M.; DESHON Jr., R.E. Effects of artificially altered foliar nitrogen levels on development and survival of young instars of western spruce budworm, Choristoneura occidentalis Freeman. Journal of Applied Entomology, v. 104, p. 121-130, 1987.

CHABOUSSOU, F. Plantas doentes pelo uso de agrotóxicos: a teoria da trofobiose. 2 ed. Porto Alegre: L\&PM, 1987. 256 p.

COMISSÃO DE FERTILIDADE DO SOLO RS/SC. Recomendações de adubação e de calagem para os estados do Rio Grande do Sul e de Santa Catarina. 3 ed. Passo Fundo: SBCS Núcleo Regional Sul, 1994. 224 p.

GALLO, D.; NAKANO, O.; SILVEIRA NETO, S.; CARVALHO, R.P.L.; BATISTA, G.C.; BERTI FILHO, E.; PARRA, J.R.; ZUCCHI, R.A.; ALVES, S.B.; VENDRAMIM, J.D. Manual de entomologia agrícola. São Paulo: CERES, 1988. 649 p.

GONÇALVES, P.A.S. Determinação de danos de Thrips tabaci Lind. em cutivares de cebola. Pesquisa Agropecuária Brasileira, Brasília, v. 31, n. 3, p. 173-179, 1996.

GONÇALVES, P.A.S. Determinação de nível de dano econômico de Thrips tabaci Lind., na cultura da cebola. In: CONGRESSO BRASILEIRO DE ENTOMOLOGIA, 16., Salvador. Resumos... Salvador: SEB/EMBRAPA - CNPMF, 1997. p. 287.
GONÇALVES, P.A.S. Eficácia de inseticidas no controle de tripes em cebola, 1996. Horticultura Brasileira, Brasília, v. 16, n. 1, p. 87-89, 1998.

INSTAT. Graphpad Instat tm, versão 2.1. Graphpad Software, 1993. 1 disquete.

JOERN, A.; BEHMER, S.T. Impact of diet quality on demographic attributes in adult grasshoppers and the nitrogen limitation hypothesis. Ecological Entomology, v. 23, n. 2, p. 174-184, 1998.

MARZO, F.; AGUIRRE, A.; CASTIELLA, M.V.; ALONSO, R. Fertilization effects of phosphorus and sulfur on chemical composition of seeds of Pisum sativum L. and relative infestation by Bruchus pisorum L. Journal of Agricultural and Food Chemistry, v. 45, n. 5, p. 1829-1833, 1997. MATSON, P.A.; PARTON, W.J.; POWER, A.G.; SWIFT, M.J. Agricultural intensification and ecosystem properties. Science, v. 277, p. 504-509, 1997.

MONEGAT, C. Plantas de cobertura do solo: características e manejo em pequenas propriedades. Chapecó (SC): Ed. Do Autor, 1991. 337 p.

McCULLOUGH, D.B.; KULMAN, H.M. Effects of nitrogen fertilization on young jack pine (Pinus banksiana) and on its suitability as a host for jack pine budworm (Choristoneura pinus pinus) (Lepidoptera: Tortricidae). Canadian Journal of Forest Research, v. 21, n. 10, p. 1447-1458, 1991. OSUNA, J.A.; ARAÚJO, J.A.C.; ARAÚJO, S.M.C.; BORTOLI, S.A.; BANZATTO, D.A.; MACEDO, E.C. Danos de Heliothis zea (Boddie, 1850) (Lepidoptera, Noctuidae) sob efeito de adubos orgânico e mineral em três genótipos de mi1ho. Anais da Sociedade Entomológica do Brasil, v. 18, p. 131-138, 1989.

PATRIQUIN, D.G.; BAINES, D.; ABBOUD, A. Soil fertility effects on pests and diseases. In: COOK, H.F. \& LEE, H.C., eds. Proceedings of the Third International Conference on Sustainable Agriculture. Londres: Wye College Press, 1993. p. 161-174.

PHELAN, P.L. Soil-management history and the role of plant mineral balance as a determinant of maize susceptibility to the european corn borer. Entomological Research in Organic Agriculture, v. 15, n. 1-4, p. 25-34, 1997.

POPP, M.P.; KULMAN, H.M.; WHITE, E.H. The effect of nitrogen fertilization of white spruce (Picea glauca) on the yellow-headed spruce sawfly (Pikonema alaskansis). Canadian Journal of Forest Research, v. 16, n. 4, p. 832-835, 1986. POWER, A.G. Influence of plant spacing and nitrogen fertilization in maize on Dalbulus maidis (Homoptera: Cicadellidae), vector of corn stunt. Environmental Entomology, v. 18, n. 3, p. 494498, 1989.

PRIMAVESI, A.M. Manejo ecológico de pragas e doenças. São Paulo: Nobel, 1988. 137 p. RUTZ, C.H.; HUGENTOBLER, U.; CHI, H.; BAUMGÄRTNER, J.U.; OERTLI, J.J. Energy flow in an apple plant-aphid (Aphis pomi De Geer) (Homoptera: Aphididae) ecosystem, with respect to nitrogen fertilization. I. Life table analyses. Plant and Soil, v. 124, n. 2, p. 273-279, 1990.
SAINI, R.K.; DAHIYA, A.S.; VERMA, A.N. Field evaluation of some insecticides against onion thrips, Thrips tabaci (Lindeman, 1888) (Thysanoptera: Thripidae). Haryana Agriculture University Journal Research, v. 19, n. 4, p. 336342, 1989.

SALAS, M.L.; CORCUERA, L.J.; ARGANDONA, V.H. Effect of potassium nitrate on gramine content and resistance of barley against the aphid Schizaphis graminum. Phytochemistry, v. 29, n. 12, p. 3789-3791, 1990.

SAMPAIO, H.N.; AZEREDO, E.H.; CASSINO, P.C.R. Dinâmica populacional do pulgão Myzus persicae (Sulzer, 1776) (Homoptera, Aphididae) no agroecossistema quiabeiro (Hibiscus esculentus L.) influenciada pelos teores de "N" e " $\mathrm{K}$ ". In: CONGRESSO BRASILEIRO DE ENTOMOLOGIA, 17., 1998, Rio de Janeiro. Resumos... Rio de Janeiro: SEB/UFRRJ, 1998a. p. 462.

SAMPAIO, H.N.; CASSINO, P.C.R.; PINTO, J.M. Interações tróficas de Aphis gossypii (Hom. Aphididae) sob regime de adubações nitrogenada e potássica na cultura do quiabeiro (Abelmoschus esculentus, Malvaceae). In: CONGRESSO BRASILEIRO DE ENTOMOLOGIA, 17., 1998, Rio de Janeiro. Resumos... Rio de Janeiro: SEB/ UFRRJ, 1998b. p. 463.

SAS INSTITUTE. SAS, versão 6.12. SAS Institute INC. Cary, NC, USA, 1996. 1 CD-ROM. SCHUCH, U.K.; REDAK, R.A.; BETHKE, J.A. Cultivar, fertilizer, and irrigation affect vegetative growth and susceptibility of chrysanthemum to western flower thrips. American Society for Horticultural Science, v. 123, n. 4, p. 727-733, 1998.

SILVA, P.R.R.; RODRIGUES, W.C.; SANTOS, W.S.; CASSINO, P.C.R.; PINTO, J.M. Influência da adubação nitrogenada e potássica sobre a população de Phyllocnistis citrella (Lep., Gracillariidae) em tangerina poncã. In: CONGRESSO BRASILEIRO DE ENTOMOLOGIA, 17., 1998, Rio de Janeiro. Resumos... Rio de Janeiro: SEB/UFRRJ, 1998. p. 459.

VANDERMEER, J. The ecological basis of alternative agriculture. Annual Review of Ecology and Systematics, v. 26, p. 201-224, 1995.

VOS, J.G.M.; FRINKING, H.D. Nitrogen fertilization as a component of integrated crop management of hot pepper (Capsicum spp.) under tropical lowland conditions. International Journal of Pest Management, v. 43, n. 1, p. 1-10, 1997.

YEPSEN Jr., R.B. Organic plant protection: a comprehensive reference on controlling insects and diseases in the garden, orchard and yard without using chemicals. Emmaus, Pennsylvania: Rodale Press, 1977. 688 p.

WHITE, T.C.R. The abundance of invertebrate herbivores in relation to the availability of nitrogen in stressed food plants. Oecologia, v. 63, p. 90105, 1984. 